\title{
Dynamic Deformation Measurement by the Sampling Moiré Method from Video Recording and its Application to Bridge Engineering
}

\author{
S. Ri ${ }^{1}\left(\mathbb{D} \cdot\right.$ H. Tsuda ${ }^{1} \cdot$ K. Chang ${ }^{2} \cdot$ S. Hsu ${ }^{2} \cdot$ F. Lo ${ }^{2} \cdot$ T. Lee $^{3}$
}

Received: 25 July 2019 / Accepted: 5 December 2019 / Published online: 10 January 2020

(C) The Author(s) 2020

\begin{abstract}
Measuring accurate dynamic deformation distribution for large-scale structures inexpensively and efficiently is a crucial challenge of structural health monitoring. In this study, a simple technique for measuring the deflection and vibration frequency from a captured video, based on the sampling Moiré method is developed. The striking advantage over conventional measurement using sensors and other imaging techniques are that the developed technique allows accurate measurement of two-dimensional deformations at multiple locations as well as the natural frequency of the target structure. Joint Photographic Experts Group (JPEG)-formatted images encoded from the recorded video with Motion-JPEG (MJPEG) format reduced the file size significantly without degrading the measurement accuracy and applied to deformation measurement. The effectiveness of the developed technique was confirmed through a field experiment of an old bridge in Taiwan. The field experiment demonstrated that both deflection and natural frequency of the bridge were successfully measured with higher $0.1 \mathrm{~mm}$ displacement accuracy and $0.05 \mathrm{~Hz}$ frequecy comparable to a conventional microwave radar displacement sensor from $20 \mathrm{~m}$ distance.
\end{abstract}

Keywords Displacement measurement $\cdot$ Phase analysis $\cdot$ Sampling Moiré $\cdot$ Image compression $\cdot$ Bridge $\cdot$ Video record

\section{Introduction}

Measuring the static and dynamic displacement of in-service structures is an important issue for design validation, performance monitoring, and safety assessment [1,2]. For the optimum design of structures and preventing unexpected accidents, and other failures, it is crucial to evaluate the displacement distribution of the target structures in service. Notably, the displacement and vibration frequency information are useful to check the soundness of infrastructures such as bridges, tunnels, and high-building [3]. Besides, a long-term measurement

\section{S. Ri}

ri-shien@aist.go.jp

1 Research Institute for Measurement and Analytical Instrumentation, National Metrology Institute of Japan (NMIJ), National Institute of Advanced Industrial Science and Technology (AIST), 1-1-1 Umezono, Tsukuba 305-8568, Japan

2 Center for Measurement Standards (CMS), Industrial Technology Research Institute of Taiwan (ITRI), Bldg. 8, 321, Sec. 2, Kuang Fu Rd, Hsinchu 30011, Taiwan

3 Department of Civil Engineering, National Central University, No. 300, Zhongda Rd., Zhongli Dist., Taoyuan City 32001, Taiwan allows continuous monitoring of structures and permits safety oversight so that an immediate warning can be issued if abnormal displacement behavior is detected. The commercial sensors such as strain gages, fiber Bragg gratings (FBGs) [4, 5], microwave radar displacement sensors [6], and laser Doppler vibrometers (LDVs) [7] have been conventionally used for measuring the displacement and vibration of large-scale structures. Displacement distribution measurement requires several sensors since the conventional sensors measure at single-point, that leads problem of cost and time-consuming. Thus, the development of the full-field measurement method capable of distributed measurement is desirable.

One approach for measuring displacement distribution in full-field of large-scale structures is the use of image processing technique. For instance, Wahbeh et al. [8] presented a vision-based approach for measuring displacements of vibrating systems. They used a high-resolution digital camera and two high-resolution red light emitting diodes (LEDs) mounted on the measured targets. The center of the red LED spots was determined from nonlinear Gaussian regression curve fit. Lee and Shinozuka [9] also presented a vision-based system for remote sensing of bridge displacement by image processing. The target has four white spots with known geometry and black background. The center of four white spots was 
determined from the black and white image. In these image processing techniques, however, the accuracy in detection of the central position of spots is limited.

Digital image correlation (DIC) technique is another kind of image processing technique for measuring displacement distributions [10]. DIC technique using random or natural patterns can obtain the deformation of the surface by comparison of the digital images before and after deformation. Recently the DIC technique was applied to measure the deflection of a bridge in static test [11] and dynamic test [12-14]. This method requires no complicated optical setup. However, DIC technique has a disadvantage that it takes long analysis time to obtain high-resolution results from field experiments susceptible to random noise and variation in light intensity.

Generally, the measurement accuracy of vision-based systems depends on several parameters, e.g. camera-to-target distance, target pattern features, lighting conditions, camera mounting stability and video-processing methods. Besides, the camera calibration including the camera intrinsic matrix, extrinsic matrix, scale factor, and lens distortion is an important procedure since the accuracy of camera calibration directly influences the displacement measurement accuracy. Xu and Brownjohn [15] reported a review of machine-vision based methodologies for displacement measurement in civil structures based on approximately one hundred papers appeared to date on this subject. According to their review paper, Khuc et al. [16] investigated the measurement accuracy of a visionbased system in a laboratory and suggested an accuracy of $0.04 \mathrm{~mm}$ in a short-range distance $(<14 \mathrm{~m})$. Martins et al. [17] demonstrated the uncertainty evaluation of displacement measurement by a vision-based system on a long-span bridge monitoring test and indicated a standard measurement accuracy of $1.7 \mathrm{~mm}$ in the vertical direction.

In our previous study, a fast and accurate phase measurement technique called the sampling Moire method $[18,19]$ for measuring displacement distribution was developed. A regular grating pattern with a known pitch placed on an object to be measured is captured with a digital camera. The displacement is obtained from calculating the phase difference of the Moiré fringe before and after deformation. Unlike the visionbased approach, the sampling Moiré method does not require camera calibration and template matching. The camera intrinsic matrix and extrinsic matrix, scale factor was not used to calculate the displacement. For the sampling Moire method, the grating pitch of a repeated pattern in physical size is a measure to obtain the actual displacement amount.

Consequently, the sub-millimeter-order deflection of a large crane $10 \mathrm{~m}$ long [20] and the thermal deformation of high-temperature piping in a thermal power plant [21] was successfully measured using the sampling Moiré method. These previous studies only utilize a series of photographs sporadically taken in static test [20] or semi-static test [21]. However, images captured with a high frame rate are necessary to measure dynamic displacement and vibration frequency. Hence, a method for measuring dynamic displacement and vibration of structures using a commercial cinema camera in this study is developed.

Recently, the functions of digital cameras have been remarkably improved in association with the enhancement of resolution, quality, and frame rate of image. For instance, a commercial cinema camera (Canon, EOS-1Dx Mark II) can record $4 \mathrm{~K}$ image size $(4096 \times 2160$ pixels $)$ at 60 frames per second (fps) in Motion-JPEG (MJPEG) format. On the other hand, this progress causes a concern that the file size of the recorded image augments enormously compared to the conventional image processing technique. To overcome this problem, a useful technique where the JPEG compressed image was used to reduce the file size is proposed. If an appropriate compression rate is used, dynamic displacement can be measured with high accuracy. Simulation results indicated that the measurement accuracy showed no deterioration even if the image quality was declined by up to $20 \%$. Furthermore, it revealed that the accuracy was slightly improved with decreasing image quality in the case of the high noise level. The effectiveness of our developed method was confirmed through the application to the displacement and vibration measurement of an old bridge in Taiwan.

\section{Principle}

\section{Outline of the Sampling Moiré Method}

The basic principle of the sampling Moire method [18] is presented in Fig. 1. When a grating of pitch $p$ (in physical scale, $\mathrm{mm}$ ) is recorded by a digital camera as shown in Fig. 1(a), the recorded intensity of a cosinusoidal grating (Fig. 1(b)) with an initial phase can be presented in Eq. (1).

$$
\begin{gathered}
f(x, y)=a(x, y) \cos \left\{2 \pi \frac{x}{P}+\varphi_{0}\right\}+b(x, y) \\
=a(x, y) \cos \{\varphi(x, y)\}+b(x, y)
\end{gathered}
$$

where $a(x, y)$ and $b(x, y)$ present the amplitude of the grating intensity and the background intensity, respectively. $P$ is the recorded grating pitch as pixel unit in the image plane, and $\varphi(x, y)$ is the phase value of the grating.

After performing down-sampling (Fig. 1(c)) and intensity interpolation (Fig. 1(d)), the multiple phase-shifted fringe patterns, $f_{m}(x, y, k)$, can be obtained, expressed as

$$
\begin{aligned}
& f_{m}(x, y ; k)=a(x, y) \cos \left\{2 \pi\left(\frac{1}{P}-\frac{1}{T}\right) x+\varphi_{0}(x, y)+2 \pi \frac{k}{T}\right\} \\
& +b(x, y)=a(x, y) \cos \left\{\varphi_{m}(x, y)+2 \pi \frac{k}{T}\right\} \\
& +b(x, y),(k=0,1, \cdots, T-1)
\end{aligned}
$$


Fig. 1 Principle of the sampling Moiré method for in-plane displacement measurement. (a) A black-and-white grating pattern with pitch, $p$, overlapped with the positions of CCD pixels (orange open squares). (b) Recorded intensity at CCD pixels. (c) Four Moiré fringe patterns by thin out every four pixels with the sampling interval $T$ (as an example, $T=4$ ). These patterns differ on the choice of the sampling point with the first sampling point in the left-hand side. The process corresponds to the phase-shifting. (d) Phaseshifted Moiré fringe patterns after linear interpolation with two adjacent sampled data in (c)

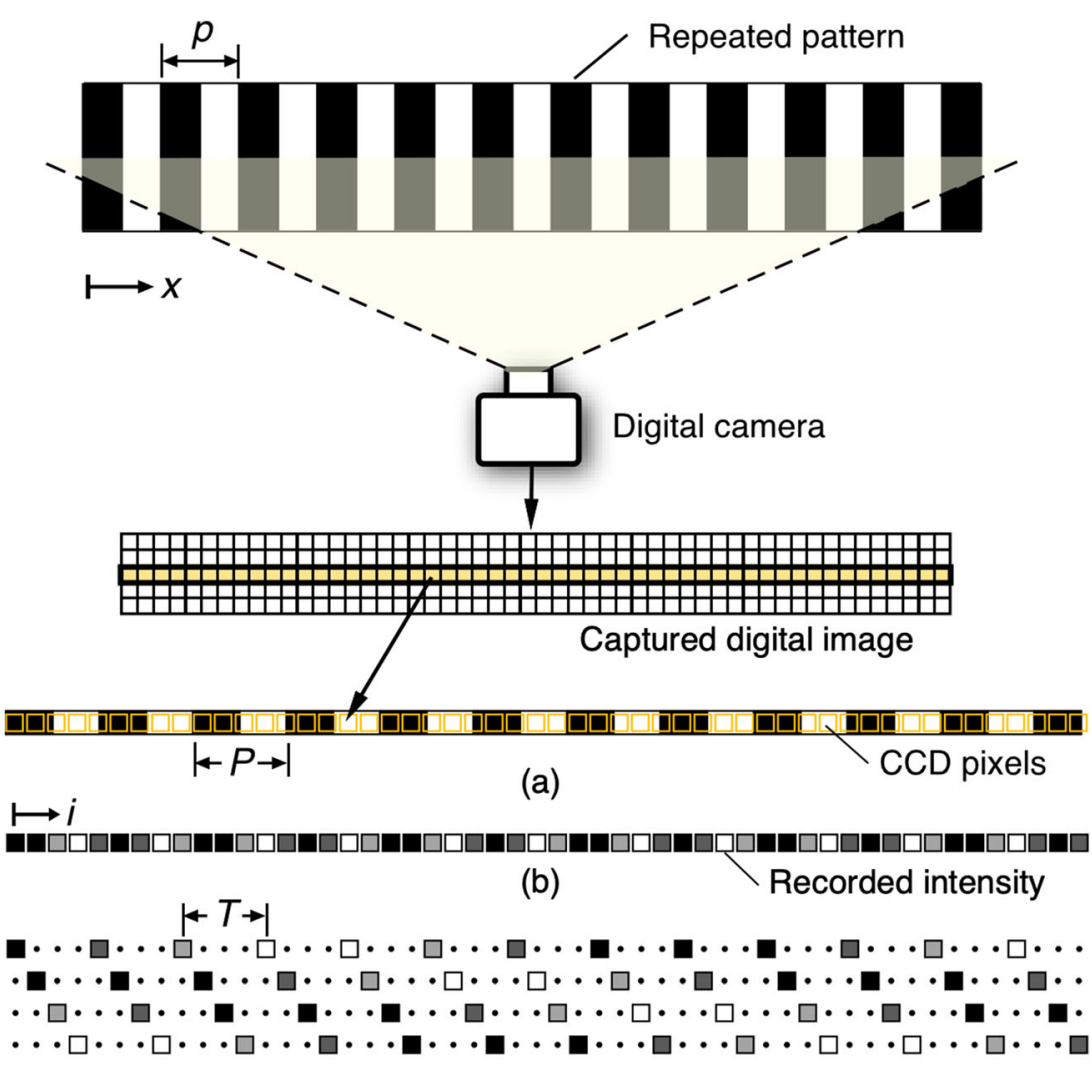

(c)

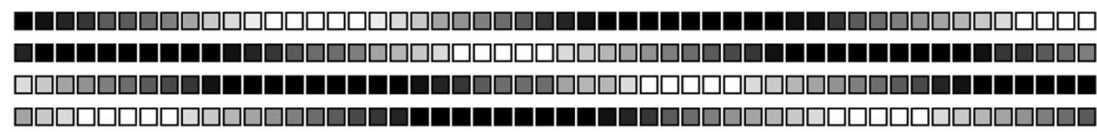
(d)

Then, the phase distribution of the Moiré fringe, $\varphi_{m}(x, y)$, can be calculated by phase-shifting method using discrete Fourier transform (DFT) algorithm, as presented in Eq. (3)

$\varphi_{m}(x, y)=-\tan ^{-1} \frac{\sum_{k=0}^{T-1} f_{m}(x, y ; k) \sin (2 \pi k / T)}{\sum_{k=0}^{T-1} f_{m}(x, y ; k) \cos (2 \pi k / T)}$

In the same manner, the phase distribution of the Moire fringe after deformation, $\varphi_{m}^{\prime}(x, y)$, can be measured. Finally, the in-plane displacement is directly obtained from the phase difference of the phase difference distribution, $\Delta \varphi_{m}(x, y)=\varphi_{m}^{\prime}(x, y)-\varphi_{m}(x, y)$, of the Moiré fringe before and after deformation.

$\delta(x, y)=-\frac{p}{2 \pi} \Delta \varphi_{m}(x, y)$

where $p$ is the grating pitch as mm unit in the physical size.

Here, the key point of the sampling Moire method is the use of the phase information of the low-frequency of a repeated pattern. Therefore, the sampling Moiré method is more robust to random noise since the high-frequency components can be easily removed by a low-pass filter.

\section{Dynamic Deformation Measurement from Video}

In this study, the conventional measurement from the sampling Moiré method using photographs was extended to the dynamic measurement using video recording. The video analysis allows us to measure dynamic deformation and the vibration of the structures.

Video images of MJPEG format can be divided into each JPEG image of time-series using FFmpeg [22] that is one of the powerful open-source libraries for handling video image. However, the huge file size of the video image compared with that of the still image leads to difficulty in data handling. Figure 2 illustrates how the file size of the video image is determined. The video image size is represented by the multiplication of image size, image quality, frame rate, and recording time. Because image size, frame rate, and recording time are determined from the specification of the camera employed and the conditions of the experiment, only image quality can be adjusted to diminish the file size. Focusing on the principle of JPEG compression, an approach to obtain an image of a smaller file size without losing measurement accuracy is proposed in this study. 


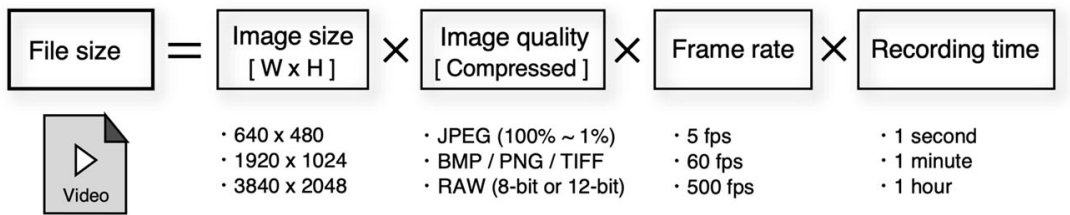

Fig. 2 The block diagram of the file size of video recording. The file size depends on the combination of the image size, the image quality, the frame rate, and the recording time. A large image size, high image

Besides the MJPEG format, there are Motion JPEG, MPEG-4, H.264, and H.265 for standard video compression in multimedia. MJPEG is a video compression format in which each image frame sequence is compressed separately as a JPEG image and no temporal compression. Different from MJPEG, MPEG data is compressed spatially and temporally as well. In this study, we mainly focus on video data with an MJPEG format. The application of another video compression format such as MPEG-4, H.264, and H.265 for higher file compression is for further study.

\section{Algorithm of JPEG Compression}

As well known, the JPEG compression algorithm [23] is one of the digital image compression standards designed for compressing both full-color and monochrome images. In the JPEG algorithm, the digital image is compressed by discarding the high-frequency components in the image because the low-frequency components are more sensitive to the human eye. As well-known, JPEG compression is a widespread technique to reduce the image size for general digital cameras. The compression time can be negligible due to the advanced development of the image processing engine in the camera chip for most cases. JPEG compression is achieved in a series of steps, as illustrated in Fig. 3.

Step 1. The pixels of the whole image are grouped into blocks of $8 \times 8$ pixels for the RAW image with lossless compression.

Step 2. A discrete cosine transform (DCT) is applied to each block, which generates 1 DC (background) and $63 \mathrm{AC}$ (amplitude) components of each spatial frequency. Then, each of the $64 \mathrm{DCT}$ components is quantized by a division of the corresponding coefficient in quantization matrix whose coefficients in upper left part are equal to 1 or more, but towards the lower right part they are getting larger.

Step 3. Each of the DCT components is rounded to an integer after quantization. Then, many zeros for higher $\mathrm{AC}$ components that are close to the right bottom part of the matrix is obtained. The higher frequency quality at high frame rate for a long time recording causes the file size huge and data handling difficult

components classified by the threshold value are discarded.

Step 4. The 64 quantized frequency components are encoded by using a combination of the Runlength encoding and the Huffman coding. Finally, a JPEG image compressed to small file size is generated and saved. The image size depends on the image quality $(I Q)$.

\section{Combination of the Sampling Moiré Method and JPEG Images}

Based on the algorithm of JEPG image compression, the measurement accuracy of our method mentioned in Sec. 2.1 seems to be insusceptible to JPEG compression if the threshold is set appropriately. This is because repeated patterns used as the target marker can be presented by lower frequency components and the unnecessary higher frequency components resulting from noise can be eliminated in the process of image compression. The combination of our sampling Moiré method using repeated patterns and JPEG compressed images would suit well to displacement distribution measurement. Thus, the JPEG compressed image for measuring the dynamic displacement to save the image file size was applied.

Figure 4 shows JPEG compression with a varying threshold value. In the DCT basic function consisting of $8 \times 8$ pixels block, the top left corresponds to the DC component and the bottom right corresponds to the highest frequency component as shown in Fig. 4(a). In the sampling Moiré method, the Moiré fringe pattern by downsampling procedure against a repeated pattern is a spatially enlarged image of the original pattern. Thus, only relatively lower frequency components are used for deformation analysis in the sampling Moiré method. The DC component (frequency zero) corresponding to the background as well as higher-frequency components originated from noise can be eliminated as unnecessary information.

As shown in Figs. 4(b) and (c), setting the threshold value to retain many frequency components can produce a high-fidelity image, but the compression rate of the file 
Fig. 3 Algorithm and procedure of the JPEG image compression. The original image is compressed by a Discrete Cosine Transform (DCT) calculation and cut the high-frequency component according to a thresholding value, i.e., the compression ratio

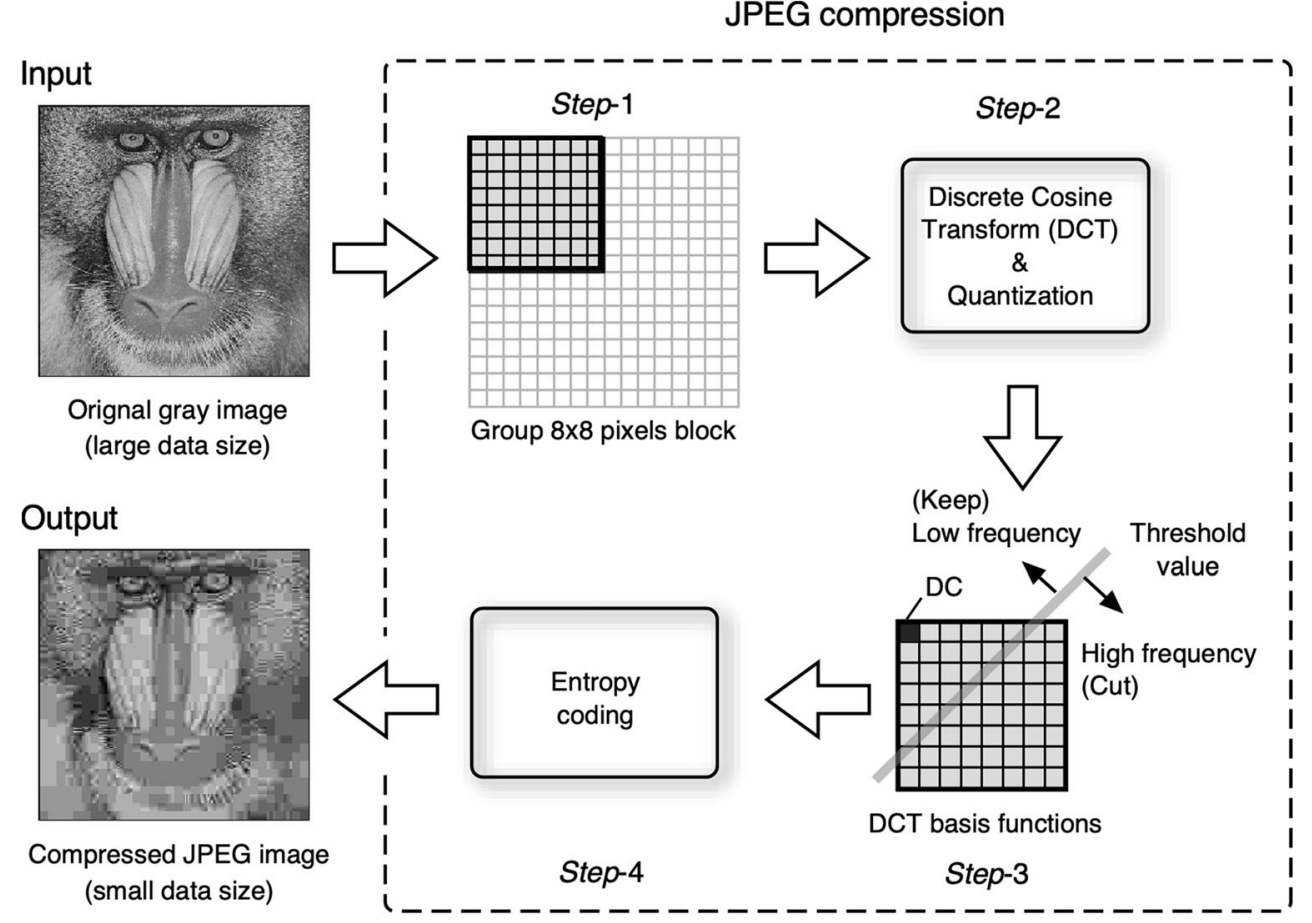

size becomes small. Conversely, higher data compression can be realized by retaining only low-frequency components. However, some low-frequency components that are required for displacement analysis in the sampling Moiré method may also be eliminated if the threshold value is set inappropriately. The setting of the thresholding value determining the image quality and size is crucial in the sampling Moiré analysis. Thus, the optimum compression ratio is explored by computer simulations and a field experiment.

\section{Simulation}

\section{Simulation Condition}

A two-dimensional grating pattern $f(x, y)$ is simulated using Eq. (1) to investigate the relationship between measurement accuracy and JPEG $I Q$. The image size of the simulated grating is $256 \times$ 256 pixels. The MATLAB "peaks" function (https://jp. mathworks.com/help/matlab/ref/peaks.html?lang=en) was added to the phase term to generate a distorted grating pattern.

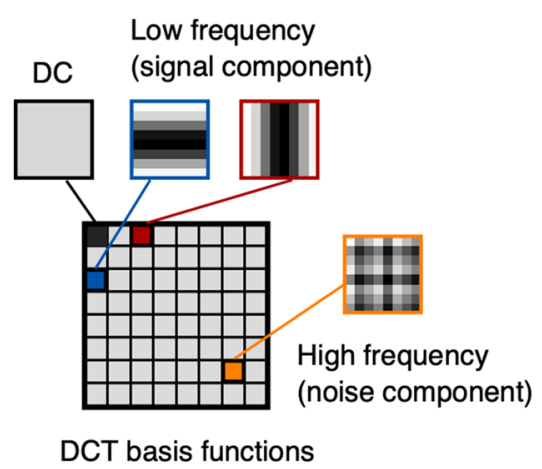

(a)

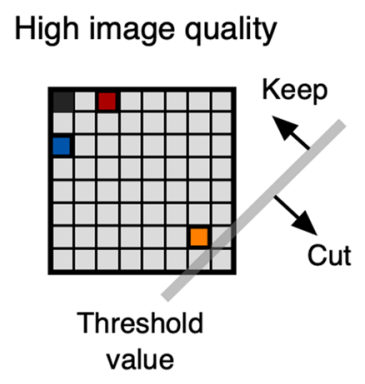

(b)

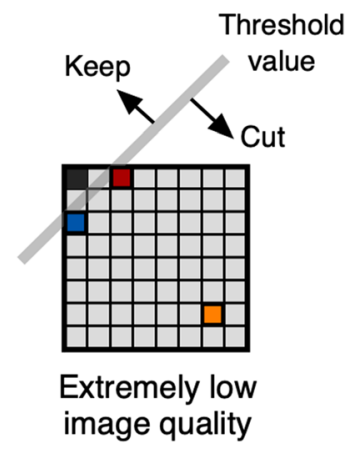

(c)

Fig. 4 Relationship between the remaining frequencies for different thresholding value (i.e., compression ratio). (a) DCT basis functions after the DCT calculation for each $8 \times 8$ pixels block. The top left corresponds to the DC component (background of the image), and the bottom right corresponds to the highest frequency component (unwanted

noise for the SM). The low-frequency component (useful signal for the $\mathrm{SM}$ ) is near the DC component. (b) High image quality for low image compression, and (c) Extremely low image quality for high image compression by changing the thresholding value 
$f(x, y)=255\left\{0.5+0.3 \cos \left(2 \pi \frac{x}{P}+\right.\right.$ peaks $\left.)+\operatorname{noise}(x, y)\right\}$

where $x, y$ are the horizontal and the vertical coordinates in the image plane, $P$ is the grating pitch in the $x$-direction, and noise $(x, y)$ is the additive random noise. Three different grating pitches of $6.1,8.1$, and 10.1 pixels are specified and the corresponding down-sampling pitches $T$ are set to 6,8 , and 10 pixels, respectively. We assume the noise level $(N L)$ has a mean of zero with three different standard deviations of 0 , 10 , and $20 \%$. Besides, images are compressed with eleven different $I Q$ s of 100, 90, 80, 70, 60, 50, 40, 30, 20, 10 and $5 \%$ for an original bitmap (BMP) grating image. The mean absolute error (MAE), the maximum (MAX) and the root mean square (RMS) phase error between the theoretical phase and the analyzed phase in the central area with $200 \times 200$ pixels were evaluated.

\section{Simulation Results}

Figure 5 shows the dependence of the simulated fringe pattern on IQ. The images of Figs. 5(b) and 5(c) look almost the same $I Q$ as the original BMP image of Fig. 5(a). In the case of $I Q=$ $5 \%$ as shown in Fig. 5(d), however, the compressed image is largely distorted with striking patterns of the $8 \times 8$ pixels block noise, which can degrade the measurement accuracy.

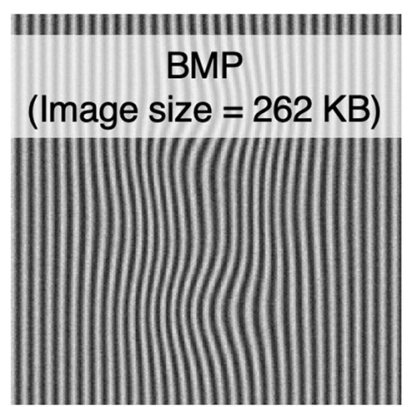

(a)

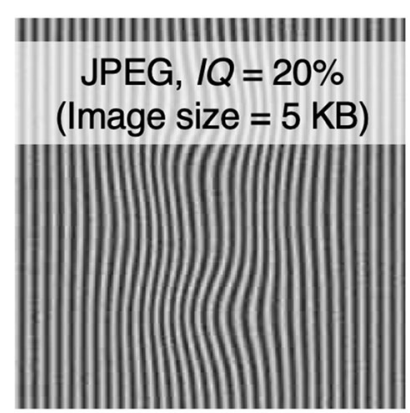

(c)

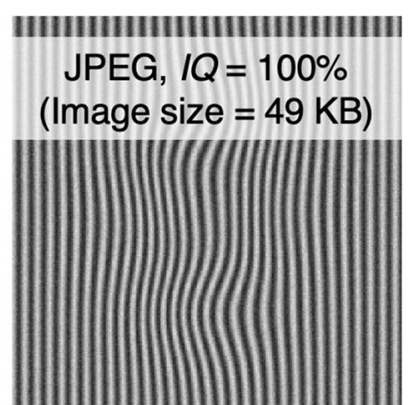

(b)

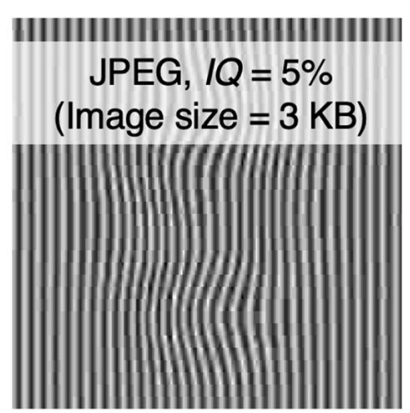

(d)
Fig. 5 Simulated fringe pattern for $P=8.1$ pixel, peaks $=1, N L=10 \%$. (a) Original bitmap (BMP) format without image compression, image quality $(I Q)$ is (b) $I Q=100 \%$, (c) $I Q=20 \%$, (d) $I Q=5 \%$
Figure 6(a) shows the relationship between the image file size and the different JPEG $I Q$ and BMP file in the case of noise level $(N L)=10 \%$. The file size of JPEG $I Q=100 \%$ (49 KB) is nearly 5 times smaller than that of the original

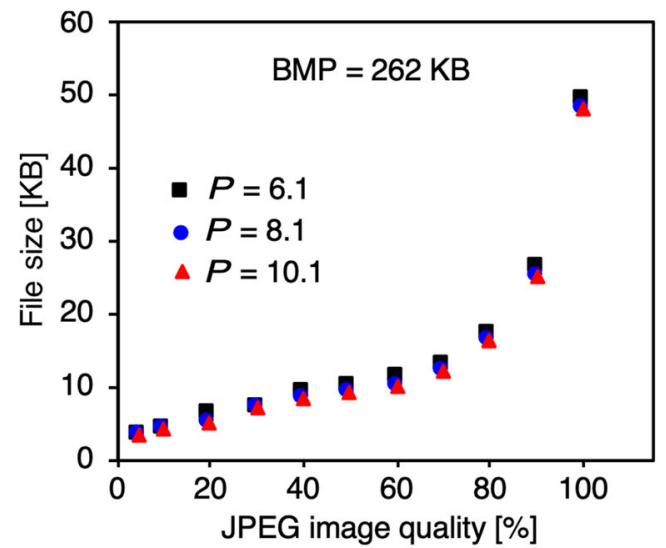

(a)

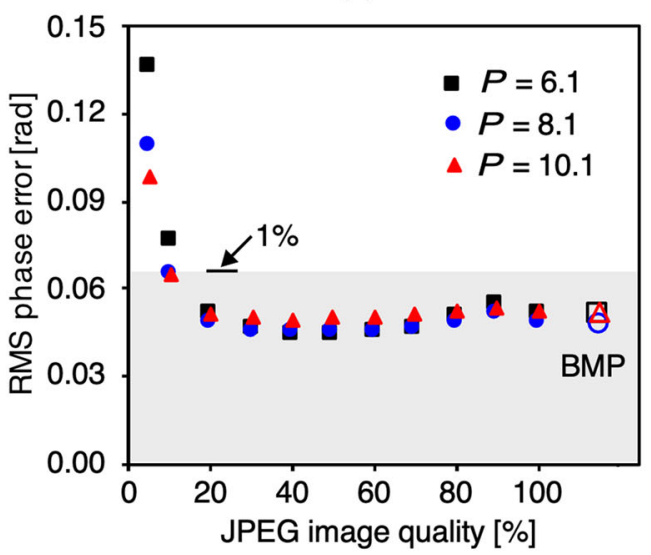

(b)

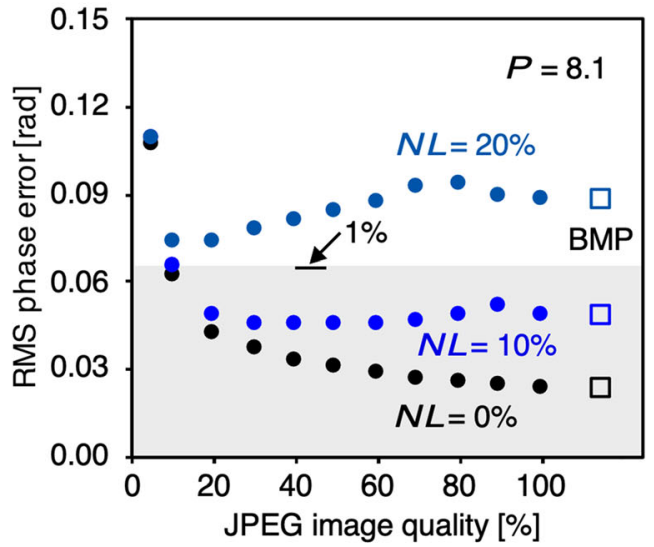

(c)

Fig. 6 Simulation results for peak=1: (a) Relationship between the image file size and the JPEG $I Q$ in the case of $N L=10 \%$ with three different pitches $P$, (b) Dependence of Root Mean Square (RMS) phase error on IQF in the case of $N L=10 \%$ with three different pitches $P$. The target domain is the central area of $200 \times 200$ pixels, (c) Dependence of the RMS phase error on $I Q$ in the case of $P=8.1$ pixels with three different noise levels. The shaded region shows RMS phase error of less than $1 \%$ of the grating pitch 
BMP image $(262 \mathrm{~KB})$ regardless of pitch size $P$. The file size of JPEG $I Q=20 \%(5 \mathrm{~KB})$ is 10 times smaller than that of $I Q=100 \%$. For the $I Q$ range below $60 \%$, the file size is approximately linearly related to JPEG $I Q$. File sizes of larger grating pitch $P$ are smaller than those of smaller $P$ for all $I Q$ range, but the file size dependence on $P$ is substantially small as compared the magnitude of file size. Thus, the influence of the grating pitch on the file size is negligible in this study.

Figure 6(b) illustrates the dependence of phase accuracy on $I Q$ in the case of $N L=10 \%$. Regardless of grating pitch $P$, the RMS phase error for the $I Q$ larger than $20 \%$ is nearly the same as that of BMP image as the reference. Notably, the measurement accuracy of JPEG $I Q=100 \%$ is very close to that of the original BMP image, even though the file size of JPEG $I Q=100 \%$ is 5 times smaller than that of BMP image. For the $I Q$ smaller than $10 \%$, the RMS phase error exceeds $1 \%$ of the grating pitch. This means that file compression with $I Q$ less than $10 \%$ is unsuitable for the present image analysis. Table 1 showed the relationship between the measurement accuracy including the MAE, MAX and RMS phase error and the JPEG image quality in the case of noise level $(N L)=10 \%$ with three different pitches $P$ in details. The trend of the MAE and MAX are similar to the RMS phase error.

Figure 6(c) depicts the sensitivity of the RMS phase error to $N L$ for $P=8.1$ pixels. In the case of $N L=0 \%$, the RMS phase error of JPEG $I Q=100 \%$ is almost the same as that of BMP image. The RMS phase error gradually increases as $I Q$ decreases. In the case of $N L=10 \%$, RMS phase error is comparable to or even smaller than that of BMP for $I Q$ larger than $20 \%$. In the case of $N L=20 \%$, RMS phase error is also comparable to or even smaller than that of BMP for $I Q$ range larger than $20 \%$ although the phase errors are larger than those of $N L=10 \%$. It is noteworthy for cases of $N L=10 \%$ and $20 \%$ that phase errors of the highly compressed image where $I Q$ ranges from $20 \%$ to $60 \%$ is somewhat smaller compared with the highest JPEG image quality of the $I Q$ of $100 \%$. However, when the $I Q$ is equal to or less than $10 \%$, the measurement accuracy is suddenly reduced since the lower frequency components that are required in the image analysis would be removed through the image compression, as illustrated in Fig. 4(c). Besides, the relationship between the measurement accuracy including the MAE, MAX and RMS phase error in case of $P=8.1$ pixels with three different $N L$ is shown in Table 2. It can be confirmed from these simulation results that the most appropriate compression rate should be $20 \%$ to save data amount without degrading the measurement accuracy.

\section{Field Experiment}

\section{Experimental Condition}

To demonstrate the effectiveness of our developed method, a field experiment for measuring the deflection of an old bridge in Tawain was carried out. Figure 7 shows the experimental setup of the tested bridge in Taiwan. The span length of the bridge is $30 \mathrm{~m}$ with a width of $7.5 \mathrm{~m}$. The distance between the camera and the bridge was about $20 \mathrm{~m}$. The Moiré marker has a two-dimensional rectangular wave pattern with the pitch size of $50 \mathrm{~mm}$. A cinema camera (Canon, 1Dc) capable of $4 \mathrm{~K}$-size $(4096 \times 2160$ pixels $)$ with a time resolution of $24 \mathrm{fps}$, and a wide-angle cinema lens (Canon, CN-E24mm T1.5 LF) of a focal length of $24 \mathrm{~mm}$ was used to record video. During the field experiment at night, the camera speed, the aperture, and the ISO were set to $1 / 30 \mathrm{~s}, \mathrm{~F} 2.8$, and 500, respectively. A microwave radar displacement sensor was also installed to measure the displacement at $1 / 2$ point of the inner beam to

Table 1 Relationship between the measurement accuracy including the mean absolute error (MAE), maximum (MAX) and root mean square (RMS) phase error and the JPEG image quality in the case of $N L=10 \%$ with three different pitches $P$

\begin{tabular}{|c|c|c|c|c|c|c|c|c|c|c|}
\hline & & \multicolumn{3}{|c|}{ Phase error(rad), $P=6.1$} & \multicolumn{3}{|c|}{ Phase error(rad), $P=8.1$} & \multicolumn{3}{|c|}{ Phase error(rad), $P=10.1$} \\
\hline & & MAE & MAX & RMS & MAE & MAX & RMS & MAE & MAX & RMS \\
\hline \multirow[t]{12}{*}{ JPEG Image quality (IQ) } & BMP & 0.039 & 0.211 & 0.050 & 0.037 & 0.207 & 0.048 & 0.041 & 0.191 & 0.052 \\
\hline & 100 & 0.039 & 0.212 & 0.051 & 0.038 & 0.211 & 0.050 & 0.041 & 0.192 & 0.052 \\
\hline & 90 & 0.042 & 0.221 & 0.054 & 0.039 & 0.243 & 0.048 & 0.041 & 0.198 & 0.053 \\
\hline & 80 & 0.038 & 0.220 & 0.049 & 0.037 & 0.223 & 0.045 & 0.040 & 0.193 & 0.052 \\
\hline & 70 & 0.035 & 0.198 & 0.045 & 0.035 & 0.195 & 0.045 & 0.039 & 0.186 & 0.051 \\
\hline & 60 & 0.034 & 0.213 & 0.044 & 0.035 & 0.175 & 0.045 & 0.039 & 0.199 & 0.050 \\
\hline & 50 & 0.033 & 0.205 & 0.044 & 0.035 & 0.169 & 0.045 & 0.038 & 0.197 & 0.049 \\
\hline & 40 & 0.033 & 0.251 & 0.044 & 0.034 & 0.173 & 0.045 & 0.037 & 0.183 & 0.049 \\
\hline & 30 & 0.035 & 0.208 & 0.045 & 0.034 & 0.196 & 0.044 & 0.038 & 0.237 & 0.050 \\
\hline & 20 & 0.039 & 0.210 & 0.051 & 0.036 & 0.225 & 0.047 & 0.038 & 0.214 & 0.051 \\
\hline & 10 & 0.058 & 0.391 & 0.076 & 0.049 & 0.252 & 0.064 & 0.049 & 0.305 & 0.065 \\
\hline & 5 & 0.101 & 0.607 & 0.135 & 0.077 & 0.509 & 0.108 & 0.072 & 0.452 & 0.098 \\
\hline
\end{tabular}


Table 2 Relationship between the measurement accuracy including the mean absolute error (MAE), maximum (MAX) and root mean square (RMS) phase error in the case of $P=8.1$ pixels with three different $N L$

\begin{tabular}{|c|c|c|c|c|c|c|c|c|c|c|}
\hline & & \multicolumn{3}{|c|}{ Phase error (rad), $\mathrm{NL}=0 \%$} & \multicolumn{3}{|c|}{ Phase error (rad), $\mathrm{NL}=0 \%$} & \multicolumn{3}{|c|}{ Phase error (rad) $\mathrm{NL}=20 \%$} \\
\hline & & MAE & MAX & RMS & MAE & MAX & RMS & MAE & MAX & RMS \\
\hline \multirow[t]{12}{*}{ JPEG image quality (IQ) } & BMP & 0.017 & 0.069 & 0.023 & 0.037 & 0.207 & 0.048 & 0.068 & 0.335 & 0.087 \\
\hline & 100 & 0.017 & 0.072 & 0.023 & 0.038 & 0.211 & 0.048 & 0.068 & 0.340 & 0.087 \\
\hline & 90 & 0.018 & 0.087 & 0.024 & 0.039 & 0.243 & 0.050 & 0.069 & 0.336 & 0.089 \\
\hline & 80 & 0.019 & 0.090 & 0.025 & 0.037 & 0.223 & 0.048 & 0.072 & 0.338 & 0.093 \\
\hline & 70 & 0.019 & 0.105 & 0.026 & 0.035 & 0.195 & 0.045 & 0.071 & 0.341 & 0.092 \\
\hline & 60 & 0.021 & 0.135 & 0.028 & 0.035 & 0.175 & 0.045 & 0.067 & 0.366 & 0.087 \\
\hline & 50 & 0.022 & 0.116 & 0.029 & 0.035 & 0.169 & 0.045 & 0.064 & 0.337 & 0.084 \\
\hline & 40 & 0.024 & 0.144 & 0.032 & 0.034 & 0.173 & 0.045 & 0.062 & 0.347 & 0.080 \\
\hline & 30 & 0.027 & 0.158 & 0.036 & 0.034 & 0.196 & 0.044 & 0.059 & 0.326 & 0.076 \\
\hline & 20 & 0.031 & 0.211 & 0.042 & 0.036 & 0.225 & 0.047 & 0.056 & 0.316 & 0.073 \\
\hline & 10 & 0.047 & 0.243 & 0.061 & 0.049 & 0.252 & 0.064 & 0.056 & 0.359 & 0.073 \\
\hline & 5 & 0.077 & 0.509 & 0.106 & 0.077 & 0.509 & 0.108 & 0.079 & 0.489 & 0.108 \\
\hline
\end{tabular}

compare the measurement accuracy between the sensor traditionally employed and the sampling Moiré method. The used microwave radar displacement sensor can measure the displacement with $0.01 \mathrm{~mm}$ to $0.1 \mathrm{~mm}$ accuracy and maximum detection range of $150 \mathrm{~m}$, and the maximum detection frequency is $20 \mathrm{~Hz}$.

Figure 8(a) shows the overall view image of the tested bridge and the position of grid-pattern markers, and the analyses image $(3800 \times 350$ pixels $)$ after trimming and gray-scale conversion from the original color JPEG image is shown in Fig. 8(b). Six markers were attached to the side walls of the bridge, as shown in Figs. 8(c) and 8(d). Two makers and three markers were attached on the outer and the inner beam, respectively. One grid-pattern M1 was attached on the pier wall as the fixed point. Each grid-pattern marker was illuminated with LED light since the experiment was conducted at midnight. The videos were taken from the direction perpendicular to the bridge, as shown in Fig. 7(c). Two trucks with a total weight of 40 tons ran through the bridge in parallel at a speed of $5 \mathrm{~km} / \mathrm{h}$ or $10 \mathrm{~km} / \mathrm{h}$ in the test. The displacement during the truck passage at the points where the grid-pattern markers were attached was measured from analyzing the recorded video. We conducted a dynamic test two times under the same
Fig. 7 Experimental setup. (a) Side view, (b) Top view, and (c) Cross section of the bridge (right) and the configuration of the camera, Moiré markers and the conventional microwave radar displacement sensor. The distance between the camera and the bridge was $20 \mathrm{~m}$. The vertical displacement was measured by using a microwave radar under the bridge and a corner reflector attached on the inner beam
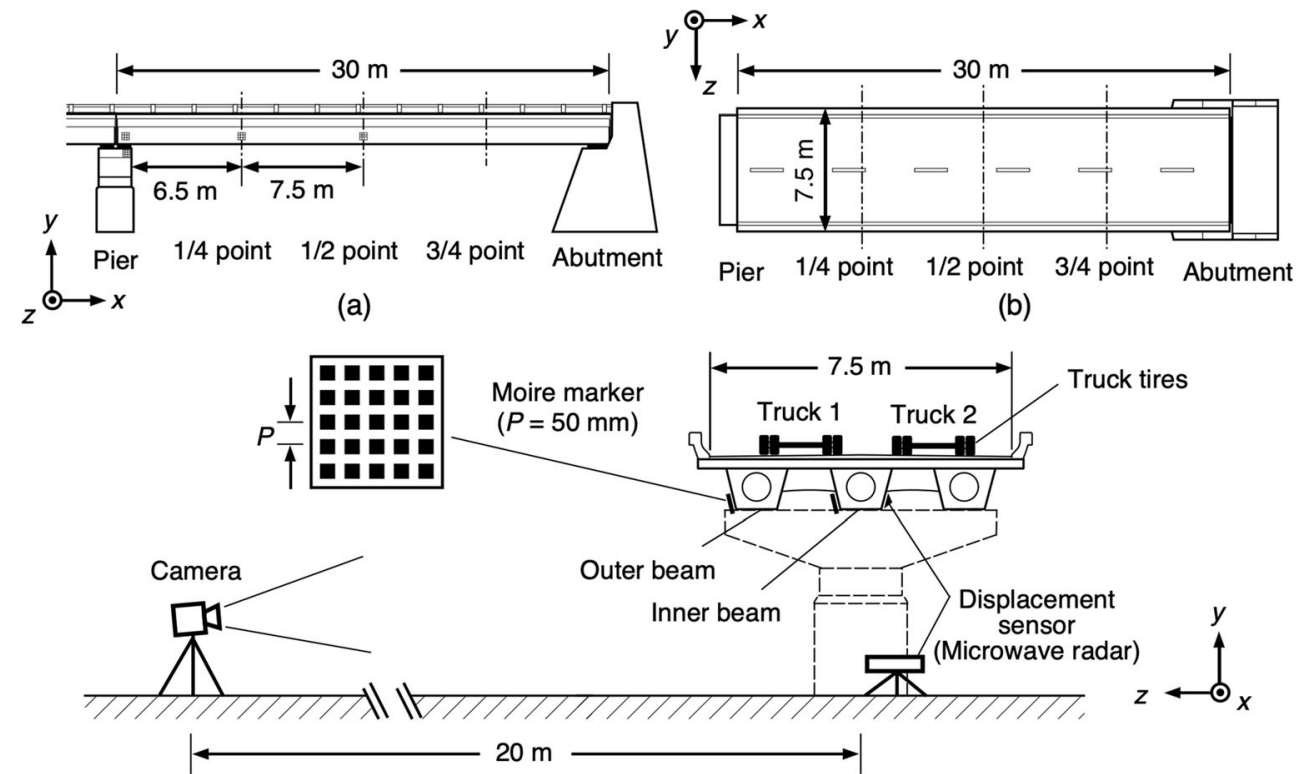

(c) 
Fig. 8 Photographs of the target bridge: (a) overall view, (b) analyses image $(3800 \times 350$ pixels) after trimming and grayscale conversion from the original color JPEG image, (c) mounting position of markers M1, M2, M3 and M4, (d) mounting position of markers M5 and M6. A corner reflector was installed on the inner beam (back of M5) to measure the deflection by the conventional microwave radar displacement sensor, as shown in Fig. 8(a) and $8(d)$

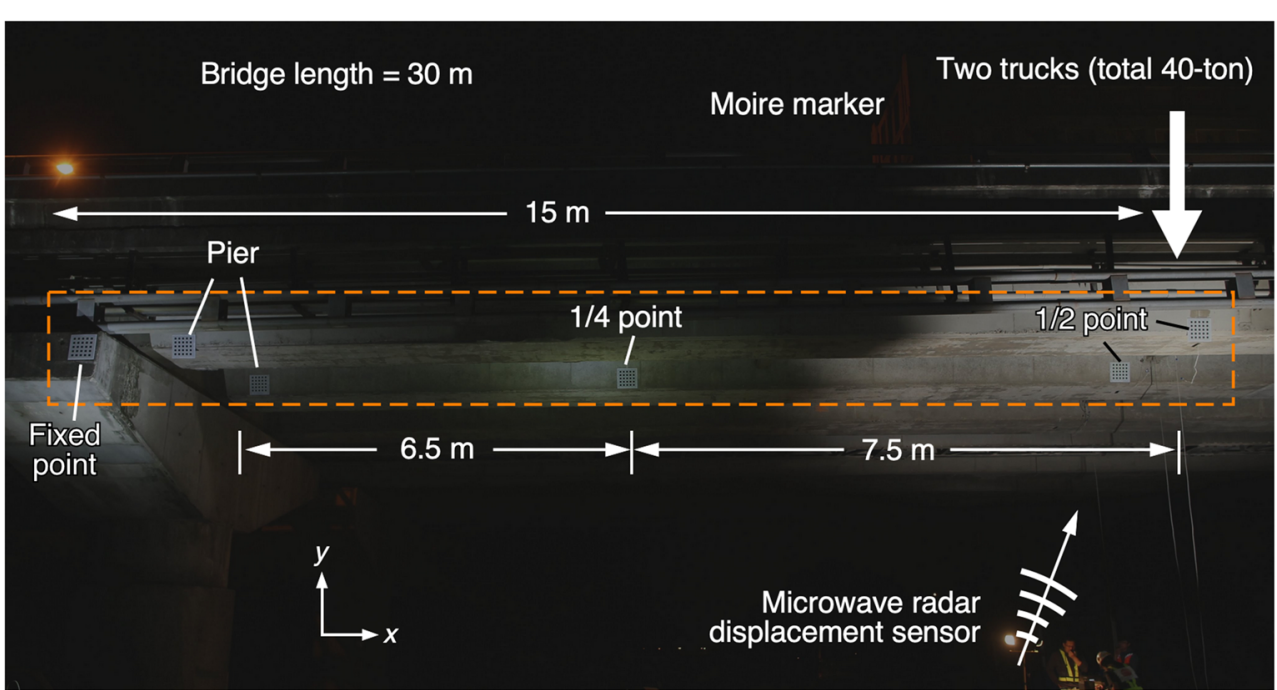

(a)

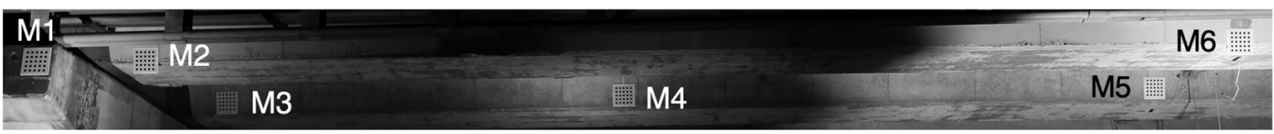

(b)

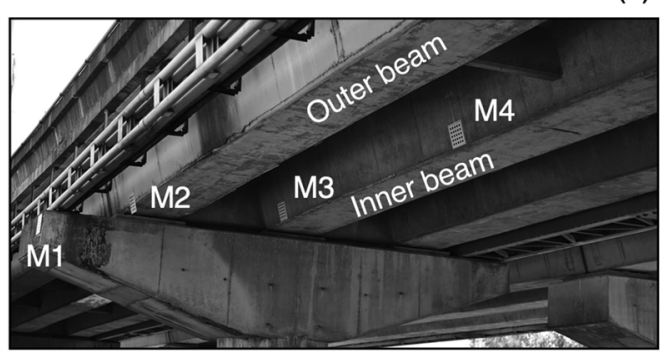

(c)

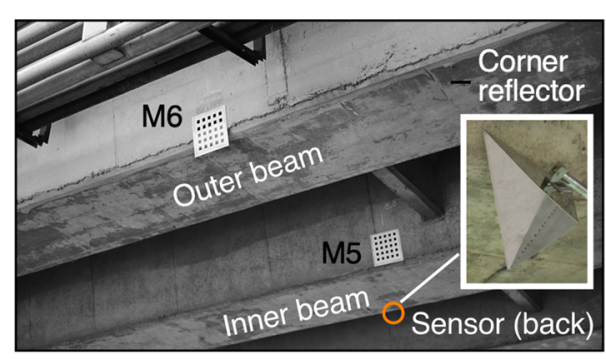

(d) condition to confirm the reproducibility of the measurements. The road was closed during the field experiment for safety and elimination of external disturbance.

Recorded movies in Motion JPEG format were converted to the colored JPEG images with a size of $4 \mathrm{~K}$ in a time series. Then, the color JPEG image was converted into a gray-scale image. The central area of the trimmed gray-scale images with $3800 \times 350$ pixels as shown in Fig. 8(b) was used to analyze deformation from the sampling Moiré method. The images recorded before the experiment were utilized as the reference to evaluate the relative displacement between each marker of M2 - M6 and marker M1 (as a fixed point) during the passage of the trucks.

Judging from the recorded image between two markers with 14-m-long (M2 and M6) and it corresponds to 3280 pixels; therefore, the spatial resolution of the recorded image was $0.234 \mathrm{pixel} / \mathrm{mm}$, and the sampling pitch $T$ was set to 12 pixels in the analysis. Figure 9 showed the image processing for a 2-dimensional rectangular grating to a 1-dimensinal cosinusoidal grating prior apply to sampling Moiré method. Figures 9(a), 9(b) and 9(c) represented the original recorded marker image, the smoothed image after SMA filter, and the extracted horizontal grating after a low-pass filter (LFP) against $x$-direction was applied. The vertical displacement pattern from the two-dimensional grating marker is extracted by applying a simple moving average (SMA) filter with a $3 \times 3$ pixel filter size, and a low-pass filter with the half-kernel size of 12 pixels and the cut-off frequency of $0.1 \mathrm{rad}$. Consequently, a cosinusoidal grating pattern in the $y$-direction can be obtained from a 2D rectangular Moiré marker. Then, the deflection can be analyzed by the sampling Moiré method as mentioned in Sec. 2.1. The average displacements

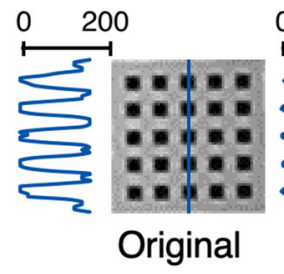

(a)

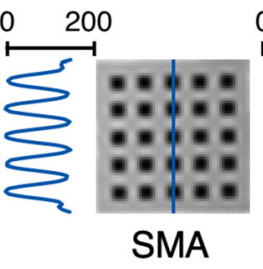

(b)

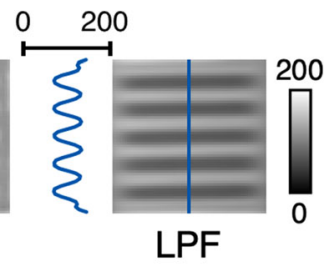

(c)
Fig. 9 Image processing of the sampling Moiré method to measure the deflection: (a) original recorded marker image, (b) smoothed image after a simple moving average (SMA) filter with a $3 \times 3$ pixels filter size, (c) extracted horizontal grating after a low-pass filter (LFP) was applied 
measured from the central region with $35 \times 35$ pixels (evaluation area) in each marker were evaluated. Besides, displacement data was processed by a smooth moving average filter using 48 data $(2 \mathrm{~s}$ period) in time-series to obtain a smooth displacement curve.

\section{Experimental Results and Discussion}

Figure 10 shows the displacement measurements of dynamic test for the truck speed of $5 \mathrm{~km} / \mathrm{h}$. Figure 10 (a) shows the deflection evaluated from the sampling Moiré method. The solid and dotted curves at $1 / 2$ point correspond to the displacement of the inner and outer beam, respectively. As expected, the displacement was maximized at $1 / 2$ point and declined in order of $1 / 4$ point and the pier. Displacement at $1 / 2$ point of the outer beam was slightly smaller than that of the inner beam. This might be caused by the smaller weight of the truck on the outer beam than that on the inner beam. Figure 10 (b) shows the displacement curve of the inner beam evaluated from the microwave radar displacement sensor. Both displacement behavior and the largest displacement measured from the conventional sensor agreed well with those measured from the sampling Moiré method. The relative difference between the largest displacement evaluated from the sampling Moiré method and the conventional sensor was only $0.04 \mathrm{~mm}$ or $0.5 \%$. The most striking advantage of the sampling Moiré method is that one-time video recording allows the displacements of multiple points with the equivalent accuracy to the conventional sensor. Another dynamic test results under the same condition are shown in Figs. 10(c) and 10(d). The difference between the largest displacement evaluated from the sampling Moiré method and the conventional sensor was $0.03 \mathrm{~mm}$. The similarity with Figs. 10(a) and 10(c) indicates high reproducibility of the experiment and the robustness of our developed method.

Figure 11 shows the deflection and vibration of the inner beam during the dynamic test at a truck speed of $10 \mathrm{~km} / \mathrm{h}$. The displacement curves without applying a smooth moving average filter shown in Fig. 11(a) include both the deflection and vibration components. The time evolution of the deflection behavior looks similar to that when the truck speed was $5 \mathrm{~km} / \mathrm{h}$ (Figs. 10(a) or 10 (c)). Displacement at $1 / 2$ point corresponded well with that evaluated from the sensor shown in Fig. 11(b).

Figures 11(c) and 11(d) show the smoothed displacement curves where vibration components are eliminated by applying a smoothed moving average filter. The displacement evaluated from the sampling Moiré method demonstrated similar
Fig. 10 Deflection measurements of dynamic test for the truck speed of $5 \mathrm{~km} / \mathrm{h}$. (a) measured $y$ directional displacement at $1 / 4$ point, $1 / 2$ point, and the pier of inner beam from the sampling Moiré method. The deflection at $1 / 2$ point of the outer beam is plotted as a broken line, (b) measured $y$-directional displacement at $1 / 2$ point of the inner beam from the conventional displacement sensor. Another dynamic test results under the same condition obtained by (c) the sampling Moiré method, and (d) the conventional displacement sensor

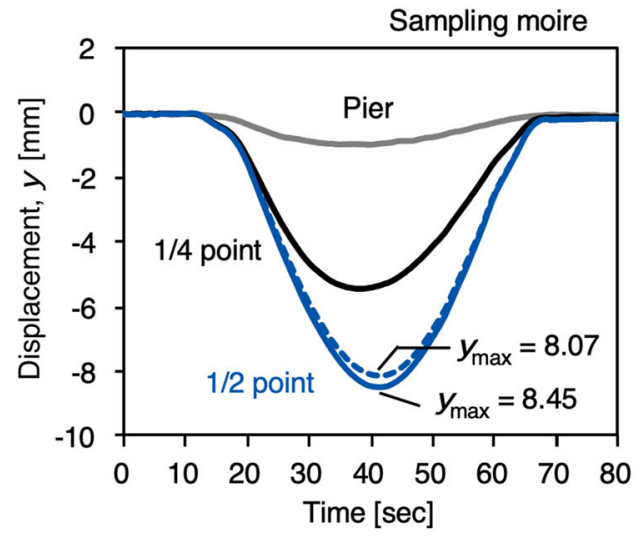

(a)

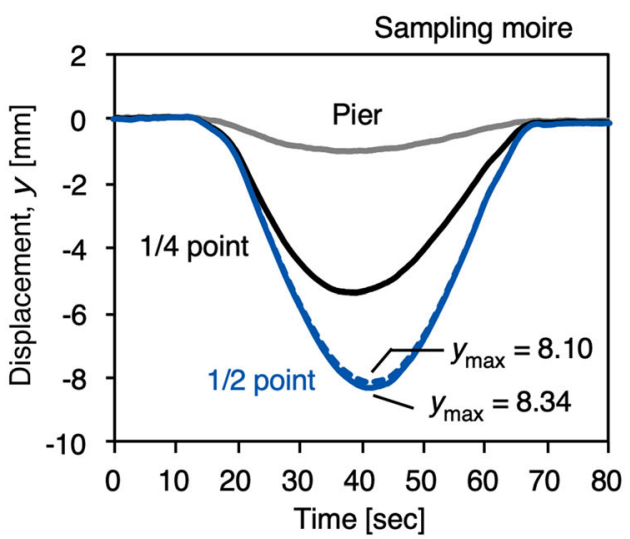

(c)

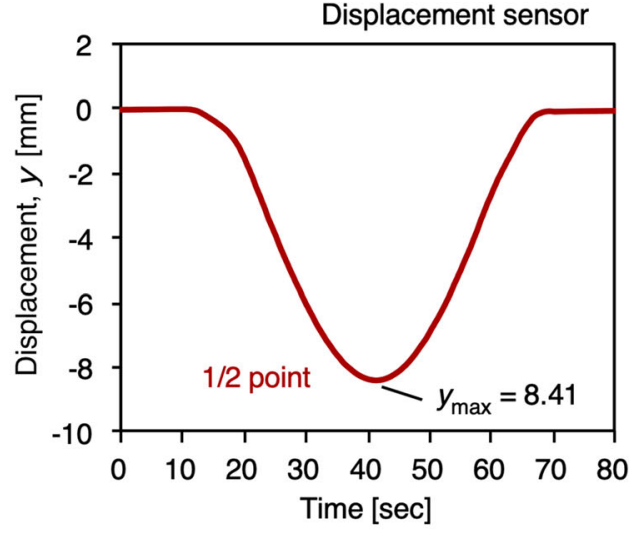

(b)

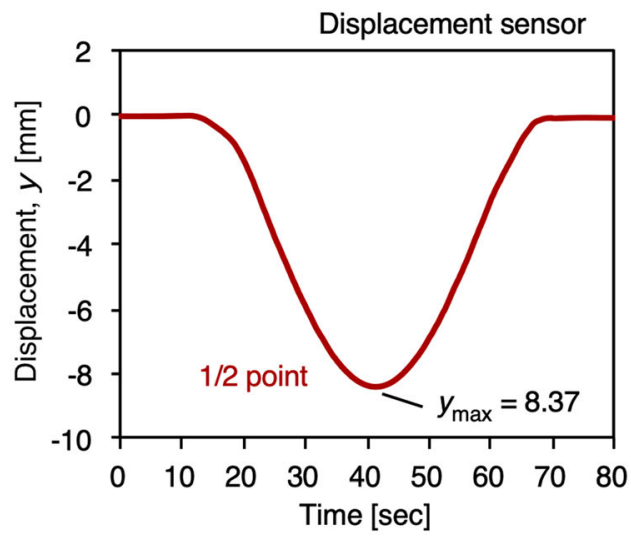

(d) 
Fig. 11 Deflection and vibration measurement results of the dynamic test of a truck speed of $10 \mathrm{~km} / \mathrm{h}$ : (a) the raw displacement curves at $1 / 4$ point, $1 / 2$ point and pier evaluated from the sampling Moiré method, (b) the raw displacement curve at $1 / 2$ point obtained with the conventional displacement sensor, (c) simple moving average (SMA) filtered displacement curves shown in (a), (d) smoothed displacement curve shown in (b), (e) and (f) vibration at $1 / 2$ point obtained by subtracting the smoothed displacement curve from the raw displacement curve, $(\mathrm{g})$ and $(\mathrm{h})$ frequency spectrum of (e) and (h), respectively

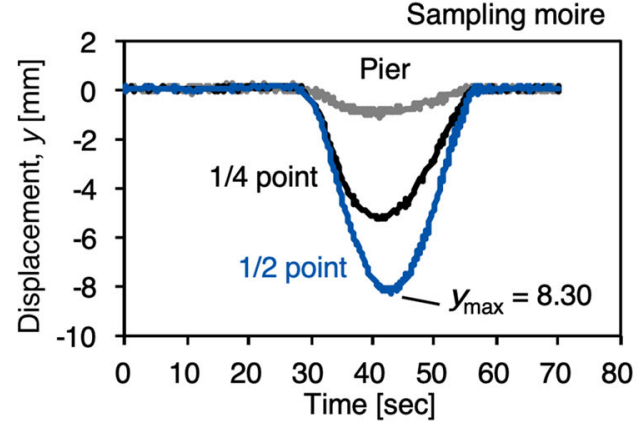

(a)

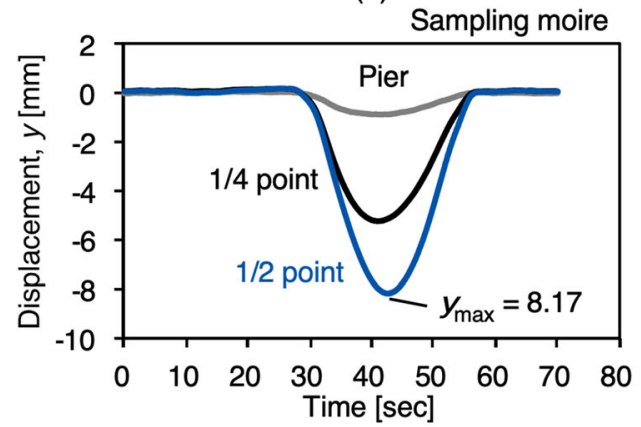

(c)

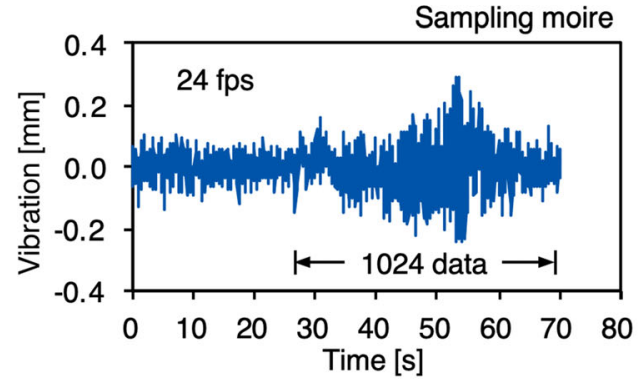

(e)

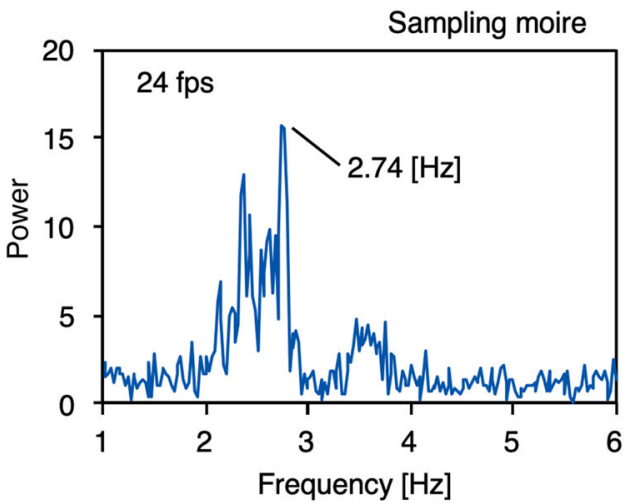

(g)

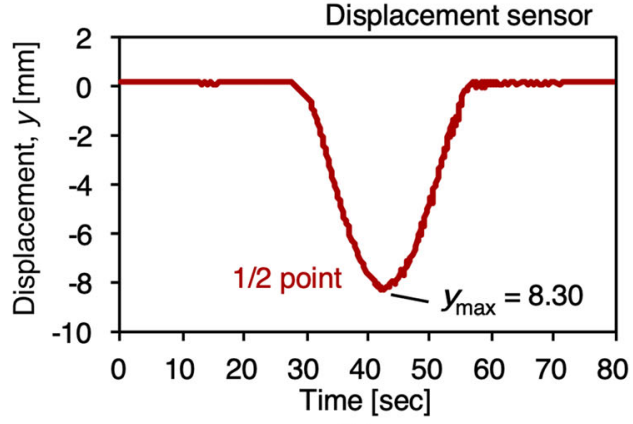

(b)

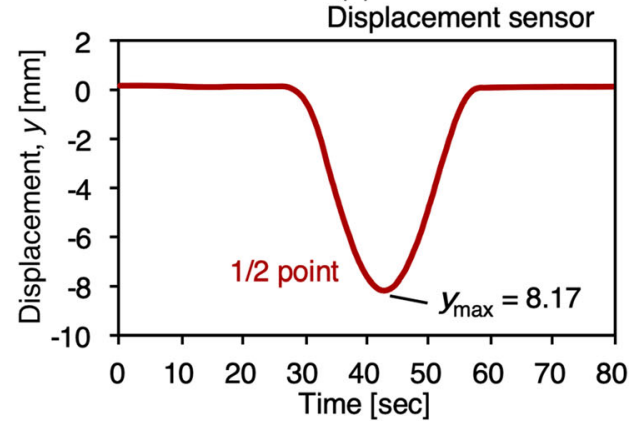

(d)

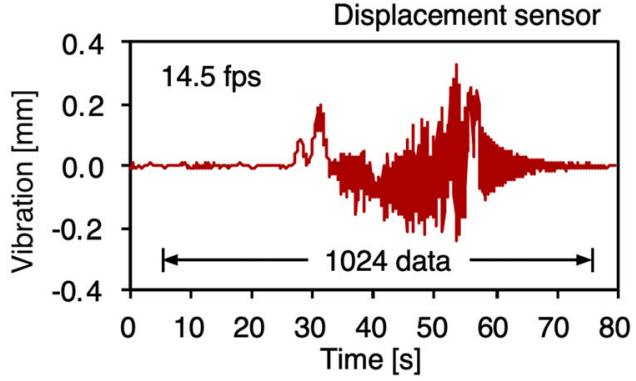

(f)

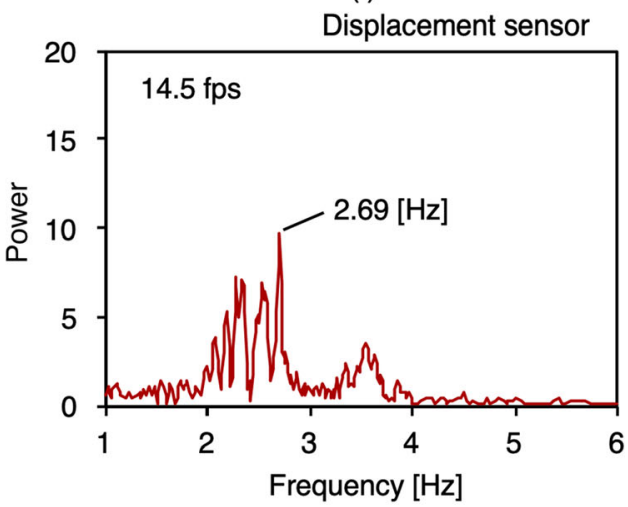

(h) behavior as observed when the truck speed was $5 \mathrm{~km} / \mathrm{h}$ shown in Figs. 10(a) or 10(c) and the maximum displacement at $1 / 2$ point corresponded with that measured from the sensor. The largest displacements evaluated from the sampling Moiré method and the conventional sensor were exactly the same to the second decimal place.
The vibration components are shown in Fig. 11(e) and 11(f) are obtained by subtracting the smoothed displacement curve from the original displacement curve. Note that in the vibration measurement from the sensor shown in Fig. 11(f), vibration is barely observed before trucks approach. A noticeable vibration of two-cycle appears at $28 \mathrm{~s}$ when the deflection 
at $1 / 2$ point starts. Then, high-frequency vibration appears from $32 \mathrm{~s}$ and continues until $57 \mathrm{~s}$ where the deflection due to truck vanishes. After that, free vibration whose intensity decreases gradually was observed. These features in vibration also are seen in the vibration curve obtained from the sampling Moiré method shown in Fig. 11(e) although high-frequency noise is superimposed throughout the measurement. The highfrequency noise is considered to correspond to error resulting from the fluctuation in image measurement because the noise has appeared since before trucks approach the measurement point. Such a measurement error seems to be inevitable in the present experiment conducted at midnight because the camera was set to a high ISO.

Frequency spectra of the vibration of 1024 data whose time period was depicted in Figs. 11(e) and 11(f) are shown in Figs. 11(g) and 11(h). Although the vibration obtained from the sampling Moiré method contained considerable noise, both vibration data have similar frequency characteristics, e.g., relatively high-frequency components ranging from 2 to $3 \mathrm{~Hz}$ and the maximum frequency component at $2.7 \mathrm{~Hz}$. The maximum frequency corresponds to the primary natural frequency of the bridge. It is noteworthy that the peak power of frequency spectra by the sampling Moiré method is greater than that by the sensor, although the higher frequency noise by the sampling Moire method is much larger than that by the sensor. This indicates that the sampling Moire method is more useful to detect the natural frequency of bridges despite large high-frequency noise. In the case of the sampling Moire method, vibration is defined from the relative displacement between $1 / 2$ point and the pier. On the other hand, the conventional sensor measures the vibration from a distance between the $1 / 2$ point and the ground that might lead to erroneous value owing to the vibration of the ground.

Fig. 12 Dynamic test results of a truck speed of $10 \mathrm{~km} / \mathrm{h}$. (a) $x$ directional displacement obtained from the sampling Moiré method (b) the average strains in the $x$ direction, (c) visualization of the measured displacement vector of the inner beam for maximum deflection
One of the significant advantages of the sampling Moiré method over conventional single-point measurement sensors is that two-dimensional displacement at multi-point can be measured using two-dimensional grid markers. Figure 12(a) shows the $x$-directional displacements along with bridge road, while Fig. 12(b) depicts the $x$-directional average strain between the $1 / 2$ point and the pier derived from the displacement shown in Fig. 12(a). As a result, the displacement vectors (i.e., two-dimensional displacement information) of each marker for maximum deflection are easily illustrated as Fig. 12(c). Such information cannot be obtained from commonly employed unidirectional displacement sensors.

In this study, the field experiment was only carried out at night due to road regulation for safety and avoid traffic congestion. The configuration of the camera for performing the field experiment in the morning or daytime is the same, except several LED lights were used at night. The variations of background conditions do not affect the measurement accuracy for the sampling Moiré method since the displacement was calculated from the phase difference of the Moire fringe. Besides, the phase of the Moiré fringe is determined for each captured image individually. Atmospheric turbulence of optical light propagating through the air is a common error source for any optical-based instrument, especially for a long-range measurement. In the case of daytime, the backlight of sunlight and the turbulences in the atmosphere would slightly deteriorate the accuracy of the sampling Moiré method. Since we experimented at night, it is reasonable to consider that errors due to the backlight of sunlight and the atmospheric turbulences during daytime are excluded in our measurements. The influence of the measurement accuracy by the atmospheric turbulence for a long-distance measure is our future work.

Our developed method is exceptionally advantageous as s deformation measurement technique featuring its simplicity,

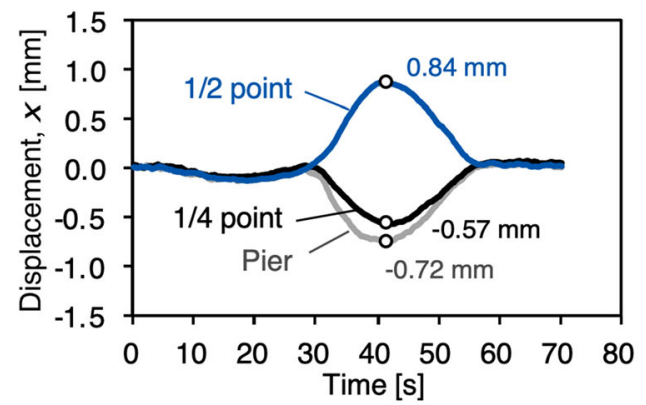

(a)

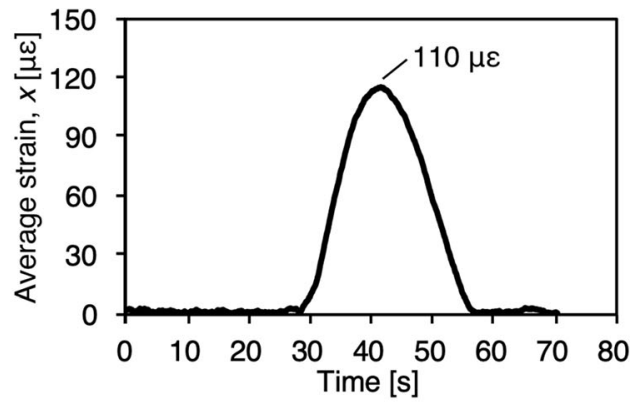

(b)

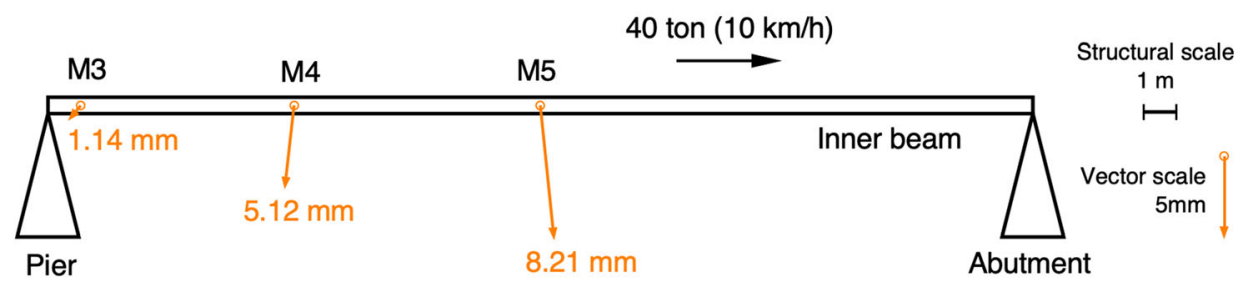

(c) 
low-cost, and high accuracy in structural health monitoring. On the other hand, a large volume of the image file is one of the obstacles in the practical application, because our proposed method utilizes time-series image data that could reach $35 \mathrm{MB} / \mathrm{s}$ at maximum. Thus, we also investigated how the $I Q$ of JPEG files influences on the accuracy of deformation measurement.

Figure 13(a) compares the image quality and file size with varying $I Q$ and the original image (top image). Figure 13(b) enlarges the repeated patterns and the random pattern of Zoom-A and Zoom-B indicated in Fig. 13(a). Though lower $I Q$ degrades the quality of the image, the feature of the image seems to be retained up to $I Q=20 \%$. In the case of $I Q=5 \%$, the feature of the random pattern can be indiscernible although the repeated pattern can be recognized. The influence of compression rate on image quality indicates that sampling Moiré method is much more robust for high compression rate than other optical methods such as DIC technique. Here, the conversion times from JPEG, $I Q=100 \%$ to JPEG, $I Q=50 \%$, JPEG, $I Q=100 \%$ to JPEG, $I Q=20 \%$, JPEG, $I Q=100 \%$ to JPEG, $I Q=5 \%$ were $0.0534,0.0534,0.0535 \mathrm{~s}$ per frame, respectively, by use of a free software called InfranVIEW operated on a Windows 7 Professional OS $(3.0 \mathrm{GHz}$ Intel Xeon CPU E5-2690 v2).

Figure 14 shows the deflection curves measured from images with different $I Q$. As shown in Figs. 14(a), 14(b), and 14 (c) whose $I Q$ is 100,50 , and $20 \%$, respectively, the deflection curves are changing smoothly. However, stepwise deflection behavior appears in Fig. 14(d) whose $I Q$ is 5\%. Here we focus on the maximum displacement, which is the most
Fig. 13 Recorded images with various image compression: (a) crop whole image ( 3800 by 350 pixels) for displacement analysis, (b) comparison between a repeated pattern (upper) and a natural random pattern (lower) in a zoom area of $100 \times 100$ pixels

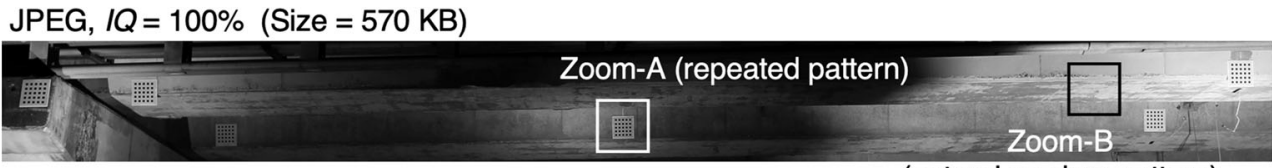

(natural random pattern)

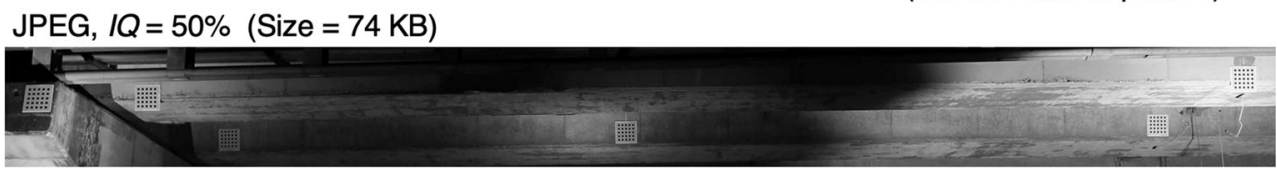

JPEG, $I Q=20 \%($ Size $=44 \mathrm{~KB})$

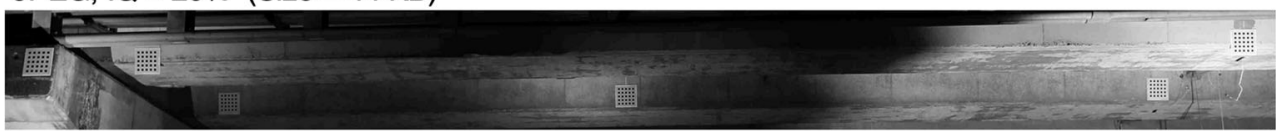

JPEG, $I Q=5 \%($ Size $=24 \mathrm{~KB})$

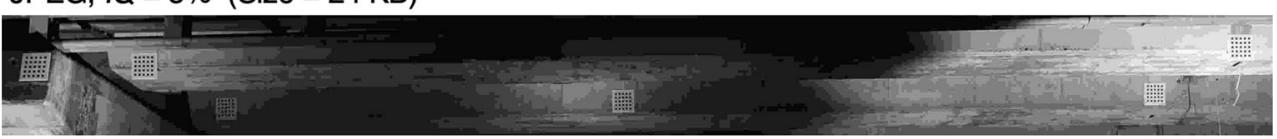

(a)

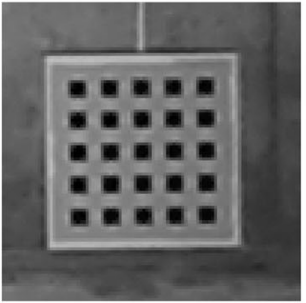

JPEG, $I Q=100 \%$

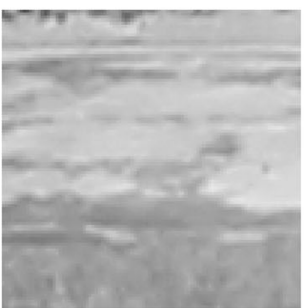

JPEG, $I Q=100 \%$

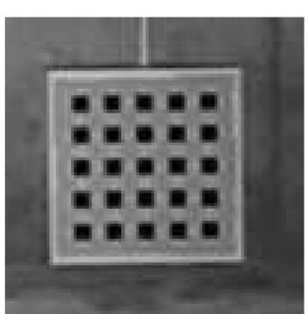

JPEG, $I Q=50 \%$

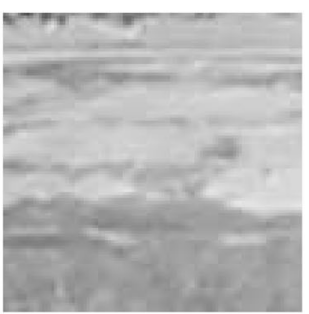

JPEG, $I Q=50 \%$

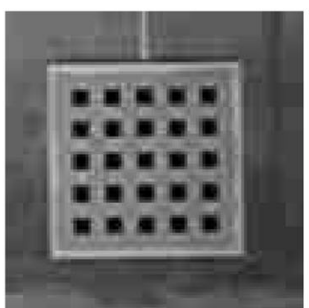

JPEG, $I Q=20 \%$

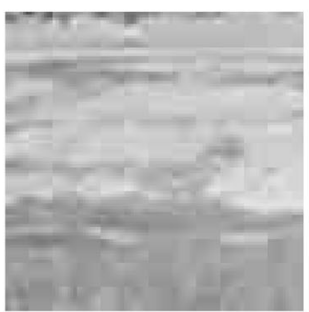

JPEG, $I Q=20 \%$

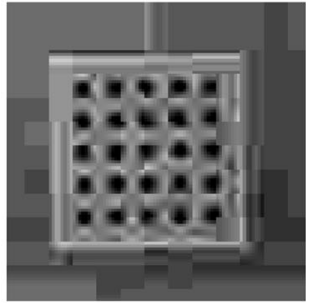

JPEG, $I Q=5 \%$

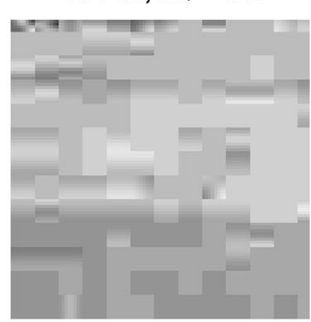

JPEG, $I Q=5 \%$

(b) 
Fig. 14 Influence of image compression rate on evaluated displacement in dynamic test at a truck speed of $5 \mathrm{~km} / \mathrm{h}$ : (a) JPEG $I Q=100 \%$, (b) JPEG $I Q=50 \%$, (c) JPEG $I Q=20 \%$, and (d) JPEG $I Q=5 \%$. The displacements at positions of pier, $1 / 4$ point and $1 / 2$ point of the inner beam are plotted

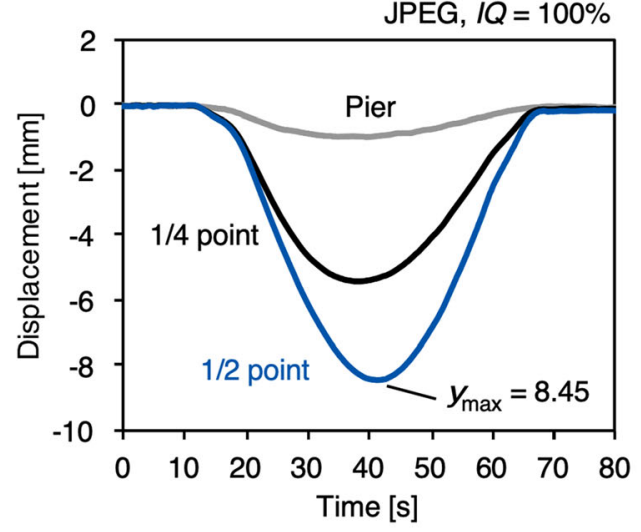

(a)

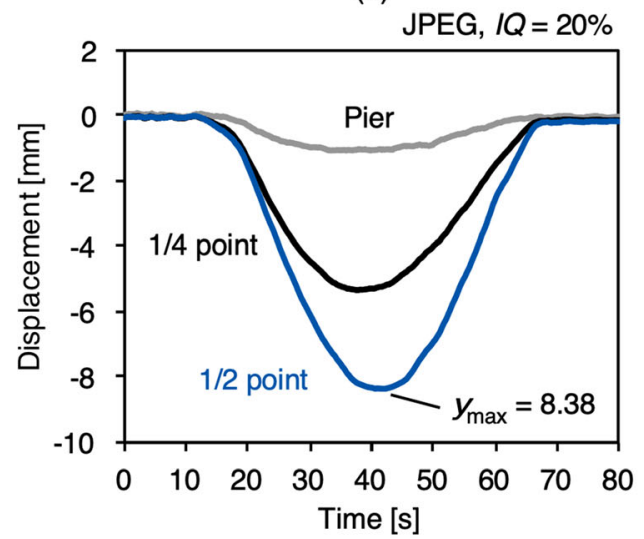

(c)

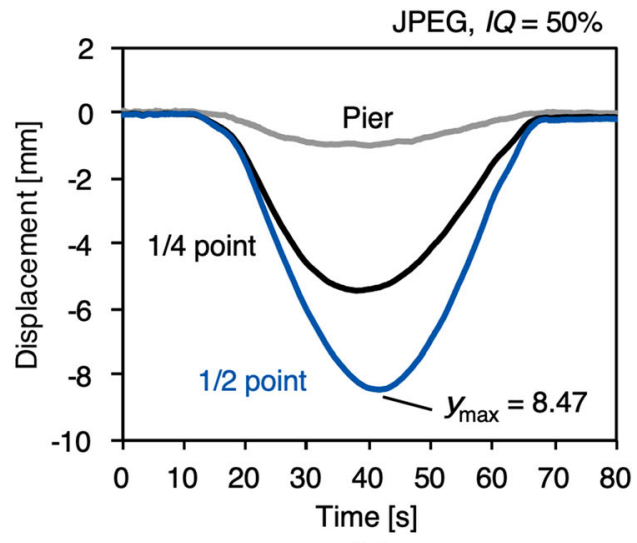

(b)

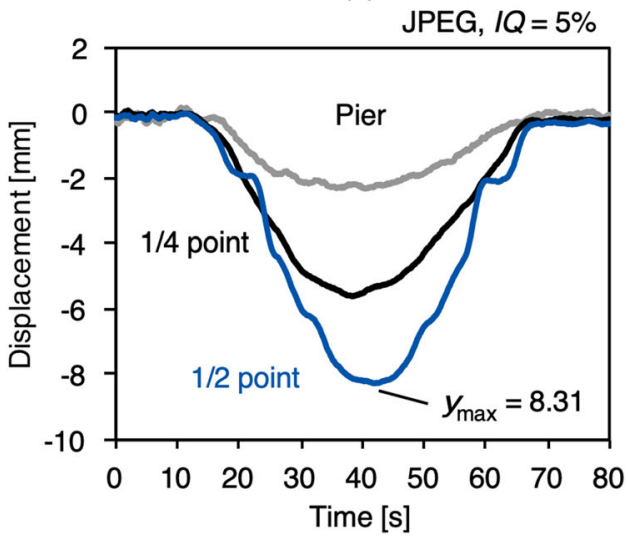

(d) important parameter in structural health monitoring of bridges. As shown in Fig. 10(b), the maximum displacement measured from the conventional sensor was $8.41 \mathrm{~mm}$. On the other hand, those obtained from the sampling Moiré method was $8.45 \mathrm{~mm}, 8.47 \mathrm{~mm}, 8.38 \mathrm{~mm}$, and $8.31 \mathrm{~mm}$ for $I Q=100,50$, 20 , and $5 \%$, respectively. Their relative errors for $I Q=100$, $50,20,5 \%$ against the maximum deflection of $8.41 \mathrm{~mm}$ measured from the conventional sensor are $0.48,0.71,-0.36$, and $-1.19 \%$, respectively. Similar to the simulation results described in Sec. 3.2, it can be confirmed that even in the case of high compression rate of $20 \%$, the sampling Moiré method measured deformation with high accuracy less than the relative error of $0.5 \%$. In the case of $I Q=20 \%$, the file size with the original image size of $570 \mathrm{~KB}$ can be reduced to $44 \mathrm{~KB}$, which is more than $90 \%$ smaller than the original size. It can be concluded from these results that our developed method is effective for measuring dynamic deformation with high accuracy even if the image files with large compression are used in the analysis.

\section{Conclusion}

In this study, an analytical method based on the sampling Moiré method is developed for measuring dynamic deformation using a video image where image data are downsized by appropriately chosen compression rate without degrading measurement accuracy. The displacement results can be obtained for any location if there are repeated patterns onto the surface of the structures in the recorded image. Applying to the deformation measurement of the bridge, our developed method is advantageous to evaluate the deformation and vibration of structures with simple procedures and high accuracy. Because there are many old concrete continuous beam bridges built on the riverbed in Taiwan, it is not easy for maintenance staff to perform on-site inspections due to terrain restrictions. Therefore, it is very suitable to measure the deflection of the bridge using the technology of this study. The main results and contributions are summarized as follows:

(1) A dynamic multi-point displacement measurement technique utilizing video data based on the sampling Moiré method was developed.

(2) The developed method can accurately measure the deflection and vibration frequency with an accuracy comparable to conventional displacement sensors.

(3) The compressed images with an appropriate compression ratio are used in this technique to reduce the image size without deteriorating the measurement accuracy. 
(4) The JPEG image quality of $20 \%$ found to be a threshold to balance the image size and measurement accuracy from the simulation and field experimental results.

(5) The developed technique was applied to deflection measurement of a bridge in Taiwan, and the effectiveness was confirmed by field experiment.

Acknowledgements We thank Dr. Shoji Kusunoki at Meteorological Research Institute in Japan for the stimulating discussion on measurement results, and Ms. Yuri Noguchi for the figure preparation.

Open Access This article is licensed under a Creative Commons Attribution 4.0 International License, which permits use, sharing, adaptation, distribution and reproduction in any medium or format, as long as you give appropriate credit to the original author(s) and the source, provide a link to the Creative Commons licence, and indicate if changes were made. The images or other third party material in this article are included in the article's Creative Commons licence, unless indicated otherwise in a credit line to the material. If material is not included in the article's Creative Commons licence and your intended use is not permitted by statutory regulation or exceeds the permitted use, you will need to obtain permission directly from the copyright holder. To view a copy of this licence, visit http://creativecommons.org/licenses/by/4.0/.

\section{References}

1. Cowing MM, Pate-Cornell ME, Glynn PW (2004) Dynamic modeling of the tradeoff between productivity and safety in critical engineering systems. Reliab Eng Syst Saf 86(3):269-284

2. Markowski AS, Mannan MS, Kotynia A, Siuta D (2010) Uncertainty aspects in process safety analysis. J Loss Prev Process Ind 23(3):446-454

3. Ri S, Tsuda H (2013) Measuring small deformations of large structures using a digital camera. SPIE Newsroom. https://doi.org/10. 1117/2.1201305.004877

4. Vohra ST, Todd MD, Johnson GA, Chang CC, Danver BA (1999) Fiber Bragg grating sensor system for civil structure monitoring applications and field tests. Proc SPIE 3746:32-37

5. Ng JH, Zhou X, Yang X, Hao J (2007) A simple temperatureinsensitive fiber Bragg grating displacement sensor. Opt Commun 273(2):398-401

6. Li C, Peng Z, Huang T, Fan T, Wang F, Horng T, Munoz-Ferreras J, Gomez-Garcia R, Ran L, Lin J (2017) A review on recent progress of portable short-range noncontact microwave radar systems. IEEE Transactions on Microwave Theory and Techniques 65(5):16921706

7. Kaito K, Abe M, Fujino Y (2005) Development of non-contact scanning vibration measurement system for real-scale structures. Struct Infrastruct Eng 1(3):189-205
8. Wahbeh AM, Caffrey JP, Masri SF (2003) A vision-based approach for the direct measurement of displacements in vibrating systems. Smart Mater Struct 12(5):785-794

9. Lee JJ, Shinozuka M (2006) A vision-based system for remote sensing of bridge displacement. NDT\&E Int 39(5):425-432

10. Sutton MA, Cheng M, Peters WH, Chao YJ, McNeil SR (1986) Application of an optimized digital correlation method to planar deformation analysis. Image Vis Comput 4(3):143-150

11. Yoneyama S, Kitagawa A, Iwata S, Tani K, Kikuta H (2007) Bridge deflection measurement using digital image correlation. Exp Tech 31(1):34-40

12. Pan B, Tian L, Song X (2016) Real-time, non-contact and targetless measurement of vertical deflection of bridges using off-axis digital image correlation. NDT\&E Int 79:73-80

13. Tian L, Pan B (2016) Remote bridge deflection measurement using an advanced video deflectometer and actively illuminated LED targets. Sensors 16:1344

14. Hoag A, Hoult N, Take W, Moreu F, Le H, Tolikonda V (2017) Measuring displacements of a railroad bridge using DIC and accelerometers. Smart Structures and Systems 19(2):225-236

15. Xu Y, Brownjohn J (2018) Review of machine-vision based methodologies for displacement measurement in civil structures. J Civ Struct Heal Monit 8:91-110

16. Khuc T, Catbas FN (2017) Completely contactless structural health monitoring of real-life structures using cameras and computer vision. Struct Control Health Monit 24:e1852

17. Martins LL, Rebordao JM, Ribeiro AS (2015) Structural observation of long-span suspension bridges for safety assessment: implementation of an optical displacement measurement system. J Phys Conf Ser 588:12004

18. Ri S, Fujigaki M, Morimoto Y (2010) Sampling moiré method for accurate small deformation distribution measurement. Exp Mech 50(4):501-508

19. Ri S, Muramatsu T (2012) Theoretical error analysis of the sampling moiré method and phase compensation methodology for single-shot phase analysis. Appl Opt 51(16):3214-3223

20. Ri S, Muramatsu T, Saka M, Nanbara K, Kobayashi D (2012) Accuracy of the sampling moiré method and its application to deflection measurements of large-scale structures. Exp Mech 52(4): 331-340

21. Ri S, Saka M, Nanbara K, Kobayashi D (2013) Dynamic thermal deformation measurement of large-scale, high-temperature piping in thermal power plants utilizing the sampling Moiré method and grating magnets. Exp Mech 53(9):1635-1646

22. FFmpeg. https://ffmpeg.org (Ver. 4.0.4, March 282019 Released)

23. Pennebaker WB, Mitchellm JL (1993) JPEG still image data compression standard. Springer Science \& Business Media, New York

Publisher's Note Springer Nature remains neutral with regard to jurisdictional claims in published maps and institutional affiliations. 